\title{
Nanoimaging Using Soft X-Ray and EUV Sources Based on Double Stream Gas Puff Targets
}

\author{
P. Wachulak*, A. Torrisi, M. Ayele, A. Bartnik, E. Węgrzyński, T. Fok, \\ J. Czwartos And H. Fiedorowicz \\ Institute of Optoelectronics, Military University of Technology, S. Kaliskiego 2, 00-908 Warsaw, Poland
}

In this work we present recent results on nanoscale imaging in the extreme ultraviolet and soft X-ray spectral ranges, describing three novel imaging systems dedicated for high spatial resolution imaging of nanoscale objects with the extreme ultraviolet and soft X-ray radiations. The extreme ultraviolet and soft X-ray full field microscopes operate at $13.8 \mathrm{~nm}$ and $2.88 \mathrm{~nm}$ wavelengths and are capable of imaging of nanostructures with a sub-50 nm spatial resolution. A soft X-ray contact microscope operates in the "water-window" spectral range from 2.3 to $4.4 \mathrm{~nm}$ wavelength, to obtain images of an internal structure of the investigated object in a thin surface layer of soft X-ray light sensitive photoresist. The development of such compact imaging systems may, in the near future, be important from the point of view of new research related to biological, material science, and nanotechnology applications. Such preliminary applications are also shown in the studies of biological samples, including carcinoma cells, diatoms, and neurons.

DOI: 10.12693/APhysPolA.133.271

PACS/topics: gas puff target, Fresnel zone plates, EUV/SXR microscopy, contact microscopy, imaging, nanometer resolution

\section{Introduction and overview of the EUV and SXR imaging methods}

Advancements in nanoscience and nanotechnology require tools and methods for nanometer scale resolution imaging. Among the methods to image a matter in the nanometer scales are extreme ultraviolet (EUV) and soft X-ray (SXR) [1] microscopies. The EUV/SXR microscopy is a tool for imaging of objects with a nanometer spatial resolution having advantages such as a possibility of obtaining additional information about the objects investigated, providing, at the same time, high optical contrast in the specific wavelength ranges used for this purpose. The main characteristics of the EUV radiation is that it is strongly absorbed in very thin layers of materials, so it provides a good optical contrast in thin films and layers of different materials. On the other hand, the SXR radiation, particularly in the so-called "waterwindow" $(\lambda=2.3-4.4 \mathrm{~nm})$, proved to be useful for high resolution imaging of biological samples, because of high optical contrast that exists between carbon and water present in the biological samples.

Laser-produced plasma sources, emitting short wavelength radiation in the SXR and EUV spectral ranges, offer an important alternative to be the drivers dedicated for compact imaging systems [2]. They allow overpassing the limited accessibility of large facilities [3], maintaining a comparable spatial resolution [4]. Over the last few years many efforts have been made to perform nanometer spatial resolution imaging in the EUV and SXR spectral

*corresponding author; e-mail: wachulak@gmail.com ranges, employing both large scale and compact sources. Using the Advanced Light source synchrotron facility a $10 \mathrm{~nm}$ spatial resolution imaging was demonstrated at $1.75 \mathrm{~nm}$ wavelength both in full-field and in scanning mode [5]. Recently, a novel approach was demonstrated, so-called dark-field X-ray microscopy, which is a non-destructive microscopy technique for the threedimensional mapping of orientations and stresses [6] that allowed for multiscale imaging with a spatial resolution of $\approx 100 \mathrm{~nm}$. Various capabilities of full-field transmission X-ray microscopy, 3D X-ray tomography, Zernike phase contrast, quantification of absorption, and chemical identification via X-ray fluorescence and X-ray absorption near edge structure imaging is now also possible with synchrotron light [7] for characterization of biomaterials.

The compact sources are being widely used for the purpose of high spatial resolution imaging in the EUV/SXR range as well. A SXR source, emitting at $\lambda=2.88 \mathrm{~nm}$, based on a liquid jet nitrogen target was employed recently for microscopy in the "water-window" range with a sub-50 $\mathrm{nm}$ spatial resolution, but this system presents a very complicated hardware [8]. Another nano-imaging technique uses compact high order harmonic generation (HHG) sources for sub-100 nm spatial resolution imaging [9]. The HHG systems, however, require a femtosecond laser as a driver for HHG process. Also, typical HHG conversion efficiency $\left(\approx 10^{-6}-10^{-4}\right)$ often results in long exposures or does not allow a proper reconstruction of the image. Ptychographic techniques, which are usually based on employment of hard X-ray beams, are possible too, providing a very high spatial resolution, but they are extensively time consuming [10]. Imaging in the EUV range permits to analyse very thin samples, nanofilms and nanostructures. This is because the EUV 
radiation is absorbed in solid materials with thicknesses of the order of $\approx 100 \mathrm{~nm}$ [11] and by gaseous materials with thicknesses of the order of a few millimetres [12]. It was demonstrated that the radiation from a capillary discharge laser, operating at a wavelength of $\lambda=46.9 \mathrm{~nm}$, permits to obtain images with a spatial resolution better than $55 \mathrm{~nm}$ [13], and that the spatial resolution of holographic images, employing the same wavelength, can be improved up to sub-50 nm [14]. A solid state target based table top EUV laser, emitting $13.2 \mathrm{~nm}$ wavelength radiation from $\mathrm{Ni}$-like $\mathrm{Cd}$ ions, was employed for imaging with a sub-38 nm spatial resolution [15]. Very nice results were also recently demonstrated by Legall et al. [16], using liquid nitrogen target based system and $1.3 \mathrm{kHz}$ repetition rate $\mathrm{Nd}$ :YAG laser, to record images of biological samples and nanostructures with a half-pitch spatial resolution of $40-50 \mathrm{~nm}$. The only drawback is quite complicated laser and target delivery system and its size comparing to other compact sources.

Another interesting and already well established technique for obtaining high resolution images of samples is projection imaging called contact microscopy. This method uses the EUV and SXR radiation, which is transmitted through the sample to expose a high resolution photoresist underneath, being in contact with the sample. Using this method, first imprints of human blood platelets, fibroblasts, and hydrated biological cells [17] were obtained.

Since there is no ideal solution for the source at the moment, it is necessary to find a good compromise in the desk-top SXR/EUV imaging systems, between the performance, namely high photon flux, possibility to obtain high spatial resolution images with low exposure time and the complexity, size, cost of these setups, which is still the main limitation of short wavelength photonbased microscopes, impeding their wide-spread. Some of these problems can be addressed employing a double stream gas puff target laser plasma source for microscopy purposes. These sources, coupled with the Fresnel zone plate (FZP) optics or as illumination sources for contact microscopy, represent a very useful technique for lab-scale analysis in order to perform high spatial resolution imaging using short wavelength radiations.

In this article we would like to present our recent activities related to the development and applications of three simple and compact SXR/EUV microscopes, capable of resolving 50-80 nm features. Those systems require short exposure times and have a desk-top footprint. The EUV/SXR source allows generating a plasma efficiently, without debris production. The microscopes are easy to operate and have compact construction. Moreover, the EUV/SXR microscopes, based on those sources [18], do not require sample preparation such as gold coating for the SEM microscopes or staining and marking, required for stimulated emission depletion (STED) microscopy. These microscopes may represent an important alternative to perform experiments in small academic laboratories on a much larger scale than with the employment of synchrotron facilities and could have a significant impact on the nanotechnology development in the near future. The goal of developing of these microscopes is to show feasibility to achieve high resolution imaging, obtain short exposure times, together with a compact footprint, which may, in the future, open a possibility for their commercialization.

\section{The description of EUV and SXR microscopes}

Full-field SXR and EUV microscopes are schematically depicted in Figs. 1 and 2, respectively, while the soft Xray contact microscope is depicted in Fig. 3. The sources for those microscopes are based on a compact Nd:YAG laser, $\lambda=1064 \mathrm{~nm}, 0.5-0.7 \mathrm{~J}$ pulse energy, 3-4 ns pulse duration and $1-10 \mathrm{~Hz}$ repetition rate. The laser beam is focused onto a double-stream gas puff target which is schematically depicted in Fig. 3a. The target is produced by an electromagnetic valve with double nozzles. Laser pulses irradiate a gaseous target producing laser plasma, which emits radiation in the EUV and SXR regions of electromagnetic spectrum. Depending on the spectral range and the type of the microscope the gas puff target source was optimized for efficient emission either in the "water window" or in the EUV spectral range. The gas pressures, nozzle position in respect to the laser focus, valve timing in respect to the laser pulse were optimized in order to maximize the photon flux at the sample plane. The inner nozzle injects a small amount of a working gas (high $Z$ gas $-\mathrm{N}_{2}$ for SXR full-field microscope, Ar for the EUV full-field microscope and contact SXR microscope), while the outer nozzle injects an outer gas (low $Z$

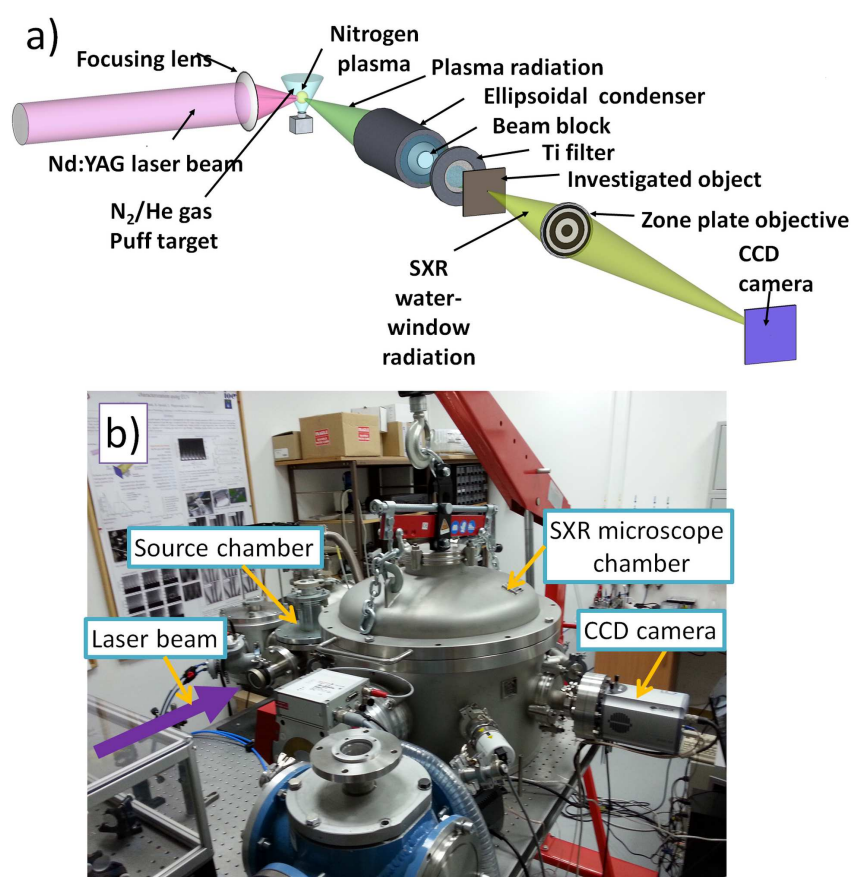

Fig. 1. (a) Scheme of the SXR full-field microscope, (b) photograph of the SXR full-field microscope. 


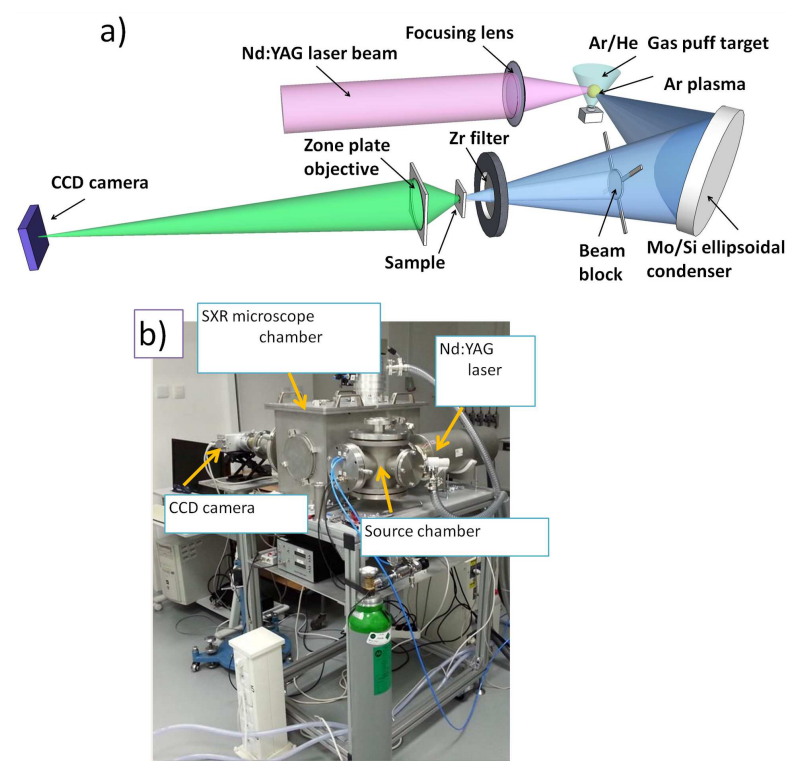

Fig. 2. (a) Scheme the EUV full-field microscope employing off-axis condenser, (b) photograph of the EUV full-field microscope.
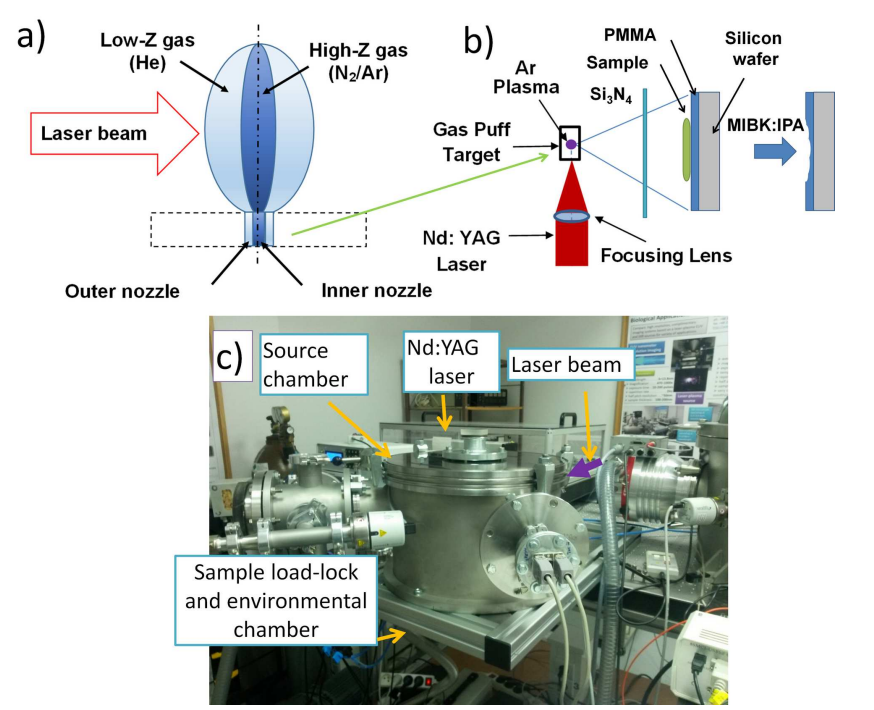

Fig. 3. (a) Scheme of the double stream gas puff target employed in laser-plasma EUV/SXR sources, (b) scheme of the contact SXR microscope, (c) photograph of the contact SXR microscope.

gas, He). The outer gas is used to narrow down the flow of the working gas, reducing its density gradient along the nozzle axis, to obtain higher target density by confining the target gas flow and, in turn, allows for higher photon yield in the EUV/SXR region.

\subsection{SXR full-field microscope}

To focus the SXR radiation from the nitrogen plasma an ellipsoidal, axi-symmetrical SXR condenser (Rigaku, Czech Republic) was used, Fig. 1a. A titanium filter (200 nm thick, Lebow), selects the He-like nitrogen line at $\lambda=2.88 \mathrm{~nm}$ from nitrogen plasma emission Filtered
SXR radiation illuminates a sample/object. A FZP objective (Zoneplates Ltd., UK) was used to form a magnified image onto a back-illuminated SXR-sensitive CCD camera (Andor, iKon-M DO-934-BN). A silicon nitride FZP (400 nm thick, $250 \mu \mathrm{m}$ in diameter, outer zone width of $30 \mathrm{~nm}$ ) with a focal length $f=2.6 \mathrm{~mm}$ at $2.88 \mathrm{~nm}$ wavelength, was used as an objective. The image plane was located $57 \mathrm{~cm}$ from the zone plate resulting in the geometrical magnification of the system of $\approx 220 \times$. Such system is capable of achieving a half-pitch spatial resolution of $\approx 6 \mathrm{~nm}[19]$. More details about the microscope can be found in [20]. The photograph of this system is depicted in Fig. 1b.

\subsection{EUV full-field microscope}

In this system, Fig. 2a, Ar plasma radiation was collected and spectrally filtered using an ellipsoidal off-axis mirror coated with Mo/Si multilayers (Reflex, Czech Republic and IOF, Germany). The condenser reflects $13.5 \pm 0.5 \mathrm{~nm}$ radiation at $45^{\circ}$ incidence angle. To eliminate longer wavelengths from the Ar plasma $(\lambda>16 \mathrm{~nm})$ a $250 \mathrm{~nm}$ thick $\mathrm{Zr}$ filter (Lebow) was used. The sample was imaged using a PMMA FZP objective (diameter $200 \mu \mathrm{m}$ and the outer zone width $\Delta r=50 \mathrm{~nm}$, Zoneplates Ltd., UK) onto the CCD camera (Andor, iKonM DO-934-BN). A geometrical magnification of the objective was $410 \times$. Such system, in this configuration, achieves a half-pitch spatial resolution of $48 \mathrm{~nm}$ [21]. More details about this system can be found in [22]. The photograph of this system is depicted in Fig. $2 \mathrm{~b}$.

\section{3. $S X R$ contact microscope}

In the final system the Ar plasma emission was tailored to the "water-window" spectral range by employing $200 \mathrm{~nm}$ thick $\mathrm{Si}_{3} \mathrm{~N}_{4}$ window. Using that filter the most energy will reside in the wavelength range from $2.8 \mathrm{~nm}$ to $4 \mathrm{~nm}$, well within the "water-window" range. The broad band SXR radiation from $\mathrm{Ar} / \mathrm{He}$ gas puff target has sufficient number of photons to expose the high resolution photoresist (500 nm thick PMMA on top of a silicon wafer) acting as a detector. The object under investigation is then placed in contact with the PMMA, Fig. $3 \mathrm{~b})$. The light that is locally transmitted by the object's structure illuminates the photoresist and changes its physical and chemical structure. After irradiation the photoresist is chemically developed (see Sect. 3.3) and modulation of the light intensity absorbed by the object is converted into a modulation of the thickness of the resist, which is later converted to an image using AFM or SEM microscopes. More details about this system can be found in [23]. The photograph of this system is depicted in Fig. 3c.

\section{Imaging with compact EUV and SXR microscopes based on a laser plasma sources}

All experimental systems mentioned in this paper were developed at the Institute of Optoelectronics, Military 
University of Technology. Those systems were also extensively used to image real samples, such us nanostructures, biological samples, organic and inorganic samples, among others. A few examples of such experiments will be presented in this section.

\subsection{SXR full-field imaging}

Two examples of "water window" images of organic samples, acquired with the full-field SXR microscope are depicted in Fig. 4. Figure $4 \mathrm{a}$ and b shows a sample of CT 26 fibroblast from Mus musculus colon carcinoma (strain BALB/c), prepared on top of a $30 \mathrm{~nm}$ thick $\mathrm{Si}_{3} \mathrm{~N}_{4}$ membrane. A direct comparison between the image acquired with a traditional optical microscope (Fig. 4a) and the SXR microscope image (Fig. 4b), acquired with $200 \mathrm{SXR}$ pulses, at a source repetition rate of $10 \mathrm{~Hz}$, exposure time of $22 \mathrm{~s}$ and detector (CCD) temperature of $-20^{\circ} \mathrm{C}$, are shown. The sample was prepared with a gradual dehydration in ethanol series (final concentration $70 \%$ EtOH), without any fixation procedure. The SXR image shows improved spatial resolution due to shorter wavelength illumination, beyond the diffraction limit of the optical, visible light microscopes. Some internal and external structures can be distinguished due to a phase contrast mechanism in the visible light microscopy images and due to a modulation in absorption of the SXR light through the sample in the SXR images. A second example consists on hippocampal neurons from E17
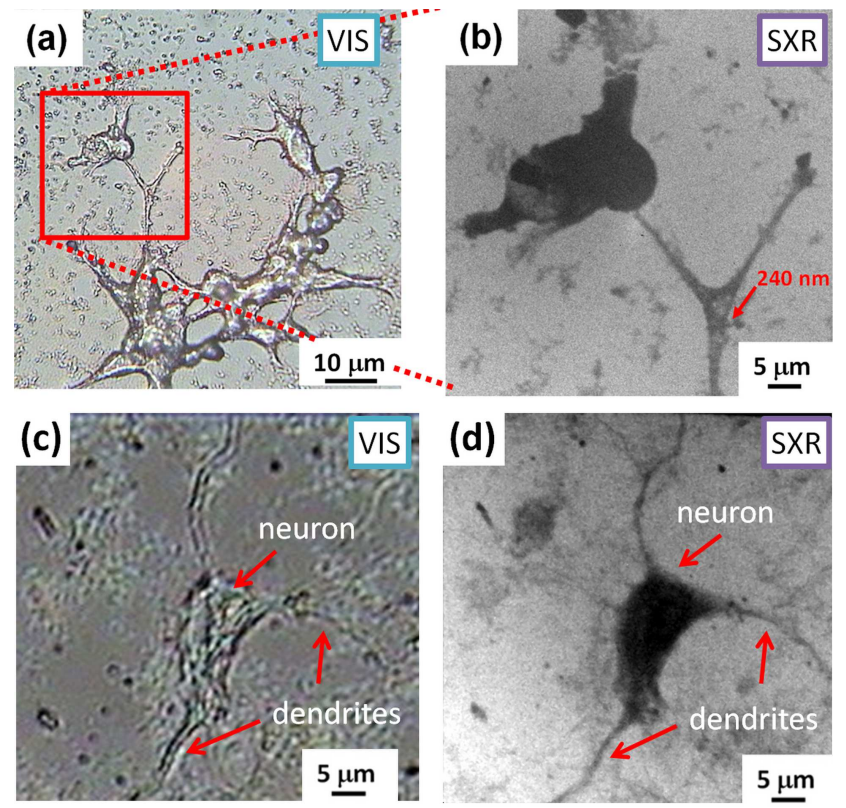

Fig. 4. CT 26 fibroblast cells. Comparison of optical image (a) and the SXR imaged (b) obtained with the "water window" microscope (red square in (a)) that shows small features of the order of $240 \mathrm{~nm}$ (indicated with red arrow). Parts (c) and (d) show a comparison of the mouse hippocampal neuron, acquired with the optical microscope $(40 \times$ objective, magnification $400 \times)$ and the SXR microscope, respectively, showing a single neuron with dendrites branching out. mouse embryos, cultured for 10 days on poly-D-lysine coated $50 \mathrm{~nm}$ thick $\mathrm{Si}_{3} \mathrm{~N}_{4}$ substrates before fixation in $4 \%$ PFA in $20 \%$ sucrose PBS, followed by dehydration from 100-70\% ethanol and air drying. The neurons were also imaged using both microscopes: visible light microscope $(40 \times$ objective, $400 \times$ magnification $)-$ Fig. $4 c$ and the SXR microscope (sample to CCD magnification of $410 \times$, exposure of 200 SXR pulses, 20 seconds) - Fig. 4d. The comparison with the optical image (Fig. 4c)) shows a significant improvement of the spatial resolution employing the "water-window" radiation. In this case it is possible to observe that the high absorption coefficient at SXR wavelengths enhances the optical contrast to the point that barely visible neuron in Fig. 4c is much better visualized in image (d). In the SXR image it is possible to distinguish the neuron and dendrites that are blurred due to inferior resolution and phase-type imaging using the visible light microscope.

\subsection{EUV full-field imaging}

In this experiment CT26 fibroblast cells, Chrysodidimus cells and diatoms were imagined using the EUV microscope and visible light microscope. The EUV images were acquired with 200 EUV pulses - 22 s exposure time and detector temperature of $-20^{\circ} \mathrm{C}$. A sample of CT 26 cells fixed with $30 \%$ hexamethildisilazane (HDMS) in absolute EtOH on top of $30 \mathrm{~nm} \mathrm{Si}_{3} \mathrm{~N}_{4}$ membrane was imaged with the visible light microscope (Vis) as depicted in Fig. 5a and at $13.84 \mathrm{~nm}$ wavelength with the EUV microscope - Fig. 5b. It can noted that the EUV im-

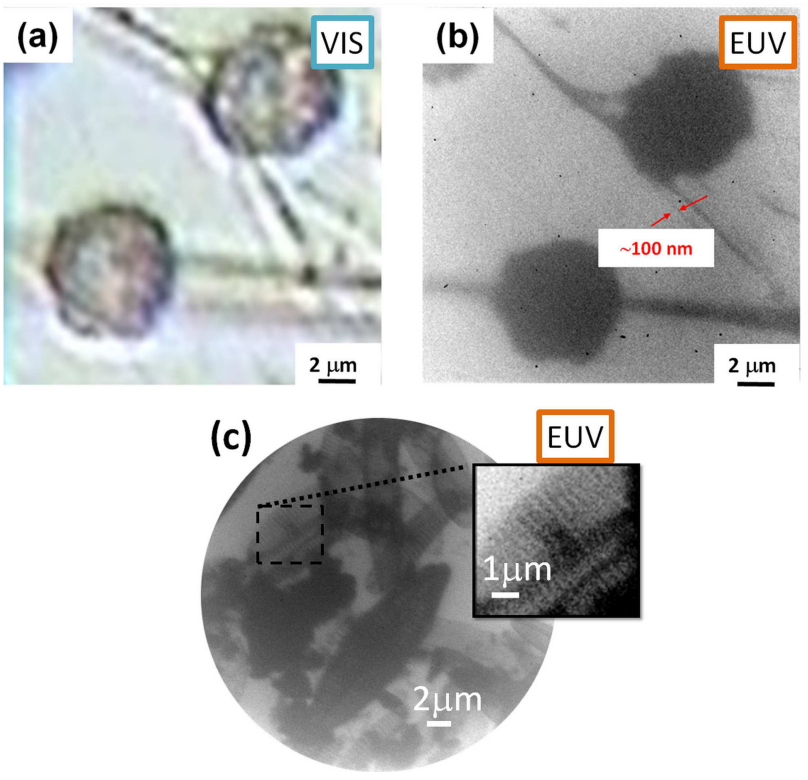

Fig. 5. Comparison of CT 26 fibroblast cells sample acquired with the optical microscope (a) and the EUV microscope (b). It is possible to appreciate small features of the order of $100 \mathrm{~nm}$ (as indicated by red arrows) in the EUV image. The EUV image of diatoms (c) obtained with exposure of 100-200 EUV pulses. Features as small as $50-100 \mathrm{~nm}$ are visible. 
age shows very high contrast and resolution enhancement and permits to investigate features of the order of $100 \mathrm{~nm}$ or smaller in size. The EUV images of diatoms deposited on top of a $30 \mathrm{~nm}$ thick $\mathrm{Si}_{3} \mathrm{~N}_{4}$ membrane are depicted in Fig. 5c, with a small inset showing diatom structures as small as a few hundreds of nanometers. The EUV images exhibit superior spatial resolution and much higher optical magnification $(410 \times)$ comparing to visible light microscopy - Fig. 5a.

\subsection{SXR contact microscopy imaging}

The contact microscope has been used for imaging of T24 (transitional cancer cell of the urine bladder, ATCC), which was prepared in collaboration with the Institute of Nuclear Physics, Cracow, Poland. The cells were grown in culture flasks (Saarstedt) in a RPMI 1640 medium (Sigma) supplemented with $10 \%$ foetal calf serum (Sigma, pH 7.4) and with 1\% mixture of antibiotics (streptomycin, neomycin, and penicillin, Sigma), at $37^{\circ} \mathrm{C}$ in $95 \%$ air $/ 5 \% \mathrm{CO}_{2}$ atmosphere. After few passages, cells were trypsinized using $0.05 \%$ in EDTA-trypsin solution (Sigma), transferred to the Petri dishes with a PMMA photoresist inside, and cultured for $24 \mathrm{~h}$. Afterwards, the cells were fixed with $3.7 \%$ paraformaldehyde solution in phosphate buffered saline (PBS, pH 7.4, Sigma). After the fixation, the cells were washed with the PBS buffer dissolved in water, and finally washed out with a deionized water. Such protocol allows drying the cells with no crystallization of salt on the sample surface.

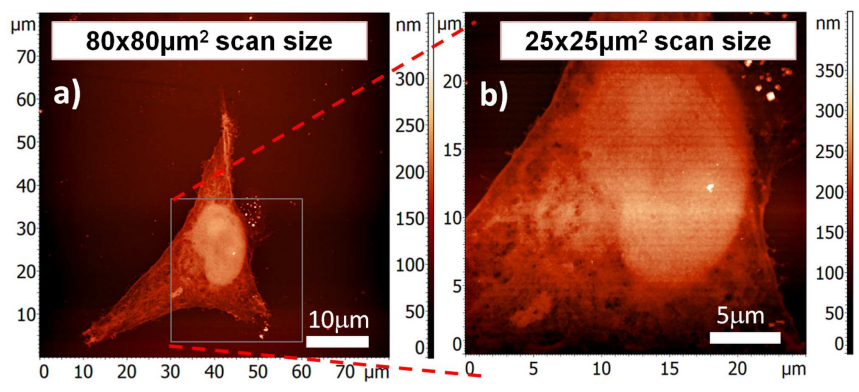

Fig. 6. SXR contact microscopy images of fixed transitional cancer cell of the urine bladder (T24), FOV $=80 \times$ $80 \mu \mathrm{m}^{2}$ (a) and $25 \times 25 \mu \mathrm{m}^{2}$ (b). Images were obtained with the SXR exposure of 200 pulses, $20 \mathrm{~s}$ and the photoresist was scanned using an AFM microscope in semicontact mode.

The contact microscope images of the T24 cell were acquired using 200 pulses of SXR radiation with exposure time of $20 \mathrm{~s}$. Following exposure to the soft X-rays, the photoresist was cleaned using a sodium hypochlorite $(5.25 \% \mathrm{w} / \mathrm{w})$ solution to remove all residues of the sample. The photoresist was then developed in methyl isobutyl ketone and isopropyl alcohol (MIBK:IPA, 1:2 $\mathrm{v} / \mathrm{v}$ ) for $120 \mathrm{~s}$. The development process result in a high resolution relief-map of the cellular structures imprinted within the photoresist, which was then viewed at a high magnification by means of an atomic force microscope (AFM, NT-MTD, Russia). The AFM scan was made in a semi-contact mode over an area of $80 \times 80 \mu \mathrm{m}^{2}$ with 512 pixels per line and $25 \times 25 \mu \mathrm{m}^{2}$ with 1024 pixels per line, respectively. The micrographs of the contact microscope images of the T24 cancer cells are shown in Fig. 6a,b. In these images, the overall structures of the cell including the central dense part (nucleus) are clearly visible with spatial resolution of approximately $80 \mathrm{~nm}$ half-pitch.

\section{Summary and conclusions}

The SXR and EUV desk-top microscopes, both fullfield and contact-type recently developed at the Institute of Optoelectronics, Military University of Technology, for imaging nanoscale objects were presented. All microscopes are based on laser plasma sources producing the EUV and SXR radiations through interaction of nanosecond laser pulses with a double stream gas puff target. The imaging systems allow capturing magnified images of the samples, with 50-80 nm half-pitch spatial resolution and exposure time as low as few seconds. Such characteristics of the microscopes may open possibility of their future commercialization. Those systems employ photons not electrons, like in the SEM, allow obtaining additional/complementary information about the sample under investigation. Their spatial resolution is comparable to what is possible to obtain with STED microscopy [24], however, such table-top systems allow for a direct acquisition of a full field images, in contrary to STED acquisition, operating in scanning mode and do not require fluorescent biomarkers or staining that can modify the morphology of the sample. Additionally, they use wavelengths providing high optical contrast, either in biological (SXR microscope) or in all matter with thicknesses less than a few hundreds of nanometers (EUV microscope).

\section{Acknowledgments}

This work is supported by the National Science Centre, Opus programme, grant agreement number UMO-2015/17/B/ST7/03718 and UMO2015/19/B/ST3/00435, the Education, Audiovisual and Culture Executive Agency (EACEA) Erasmus Mundus Joint Doctorate Programme Project No. 2012-0033 and from the European Union's Horizon 2020 research and innovation program, under Laserlab-Europe IV, grant agreement No. 654148.

We would like to thank to Andrzej Kowalik and Anna Rojek, from Institute of Electronic Materials Technology (ITME), Warsaw, Poland for the preparation of PMMA coated wafers. We also acknowledge Tomasz Kobiela and Anna Sobiepanek, from the Faculty of Chemistry, Warsaw University of Technology, and Małgorzata Lekka, from the Institute of Nuclear Physics, Krakow, Poland for providing the biological samples used for contact microscopy experiments. Additionally, we would like to express our gratitude to Tomas Parkman, Šarka Salacová and Jana Turňová from the Faculty of Biomedical Engineering, Czech Technical University in Prague, for preparation of biological samples for the EUV and SXR microscopes and to Michal Odstrcil from Paul Scherrer Institut, Switzerland, for preparation of neuron sample. 


\section{References}

[1] The International Organization for Standardization, ISO 21348 Definitions of Solar Irradiance Spectral Categories, 2007, p. 6.

[2] B. Li, T. Higashiguchi, T. Otsuka, W. Jiang, A. Endo, P. Dunne, G. O'Sullivan, Appl. Phys. Lett. 102, 041117 (2013).

[3] M.A. Le Gros, G. Mcdermott, B.P. Cinquin, J. Synchr. Radiat. 21, 1370 (2014).

[4] S. Marino, S. Palanco, M. Gabás, R. Romero, J.R. Ramos-Barrado, Nanotechnology 26, 55303 (2015).

[5] W. Chao, P. Fischer, T. Tyliszczak, S. Rekawa, E. Anderson, P. Naulleau, Opt. Expr. 20, 9 (2012).

[6] H. Simons, A. King, W. Ludwig, C. Detlefs, W. Pantleon, S. Schmidt, I. Snigireva, A. Snigirev, H.F. Poulsen, Nature Commun. 14, 6098 (2015).

[7] J.C. Andrews, F. Meirer, Y. Liu, Z. Mester, P. Pianetta, Microsc. Res. Techn. 74, 671 (2011).

[8] K.W. Kim, Y. Kwon, K.Y. Nam, J.H. Lim, K.G. Kim, K.S. Chon, B.H. Kim, D.E. Kim, J. Kim, B.N. Ahn, H.J. Shin, S. Rah, K.H. Kim, J.S. Chae, D.G. Gweon, D.W. Kang, S.H. Kang, J.Y. Min, K.S. Choi, S.E. Yoon, E.N. Kim, Y. Namba, K.H. Yoon, Phys. Med. Biol. 51, N99 (2006).

[9] J.J. Park, D.S. Kim, S.C. Jeon, K.H. Lee, J. Lee, K.N. Kim, J.J. Yoo, C.H. Nam, Opt. Lett. 34, 235 (2009).

[10] M. Dierolf, P. Thibault, A. Menzel, C.M. Kewish, K. Jefimovs, U. Schlichting, K. Von König, O. Bunk, F. Pfeiffer, New J. Phys. 12, 035017 (2010).

[11] M.C. Marconi, P.W. Wachulak, Progr. Quant. Electron. 34, 173 (2010).

[12] P.W. Wachulak, Ł. Wegrzynski, A. Bartnik, T. Fok, R. Jarocki, J. Kostecki, M. Szczurek, H. Fiedorowicz, Laser Part. Beams 31, 219 (2013).
[13] P.W. Wachulak, C.A. Brewer, F. Brizuela, C.S. Menoni, W. Chao, E.H. Anderson, R.A. Bartels, J.J. Rocca, M.C. Marconi, J. Opt. Soc. Am. B 25, B20 (2008).

[14] P.W. Wachulak, M.C. Marconi, R. Bartels, C.S. Menoni, J.J. Rocca, J. Opt. Soc. Am. B 25, 1811 (2008).

[15] G. Vaschenko, F. Brizuela, C. Brewer, M. Grisham, H. Mancini, C.S. Menoni, M.C. Marconi, J.J. Rocca, W. Chao, Opt. Lett. 30, 2095 (2005).

[16] H. Legall, G. Blobel, H. Stiel, W. Sandner, C. Seim, P. Takman, D.H. Martz, M. Selin, U. Vogt, Opt. Expr. 20, 18362 (2012).

[17] M. Kado, M. Kishimoto, S. Tamotsu, K. Yasuda, M. Aoyama, K. Shinohara, X-Ray Lasers Coher. XRay Sourc. Devel. Appl. X 8849, 88490C (2013).

[18] P.W. Wachulak, A. Bartnik, M. Skorupka, J. Kostecki, R. Jarocki, M. Szczurek, Ł. Wegrzynski, T. Fok, H. Fiedorowicz, Appl. Phys. B 111, 239 (2013).

[19] P. Wachulak, A. Torrisi, M.F. Nawaz, A. Bartnik, D. Adjei, S. Vondrová, J. Turňová, A. Jančarek, J. Limpouch, Microsc. Microanal. 21, 1214 (2015).

[20] P.W. Wachulak, A. Torrisi, A. Bartnik, D. Adjei, J. Kostecki, Ł. Wegrzynski, R. Jarocki, M. Szczurek, H. Fiedorowicz, Appl. Phys. B 118, 573 (2015).

[21] P.W. Wachulak, A. Torrisi, A. Bartnik, Ł. Węgrzyński, T. Fok, H. Fiedorowicz, Appl. Phys. B 123, 1 (2016).

[22] A. Torrisi, P. Wachulak, Ł. Węgrzyński, T. Fok, A. Bartnik, T. Parkman, S. Vondrová, J. Turňová, B.J. Jankiewicz, J. Microsc. 265, 251 (2017).

[23] M.G. Ayele, J. Czwartos, D. Adjei, P. Wachulak, I.U. Ahad, A. Bartnik, Ł. Wegrzynski, M. Szczurek, R. Jarocki, H. Fiedorowicz, Acta Phys. Pol. A 129, 237 (2016).

[24] K. Otomo, T. Hibi, Y. Kozawa, Microscopy 64, 227 (2015). 